\title{
Improvement in ionic conductivities of poly-(2-vinylpyridine) by treatment with crotonic acid and vinyl acetic acid
}

\author{
ANNA GOGOI and NEELOTPAL SEN SARMA* \\ Physical Sciences Division, Polymer Unit, Institute of Advanced Study in Science and Technology, \\ Paschim Boragaon, Garchuk, Guwahati 781 035, Assam, India
}

MS received 13 May 2014; revised 24 September 2014

\begin{abstract}
The synthesis, characterization and improved ionic conductivities of the salts of poly-(2-vinylpyridine) with crotonic acid and vinyl acetic acid are reported here. In this study, the alternating current conductivity measurements were carried out within the temperature range of $30-90^{\circ} \mathrm{C}$ and the frequency range of $1 \mathrm{~Hz}-100 \mathrm{kHz}$ in solid state. A two- to three-fold increase in conductivity was observed for vinyl acetic acid salt whereas one- to twofold increase was observed for crotonic acid salt. The ionic transport numbers of the salts were measured with the help of the Wagner polarization technique which reveals that the percentage of ionic character of the salts are significantly higher compared with the polymer. The percentage of water uptake by the polymer and its salts were also observed.
\end{abstract}

Keywords. Polyelectrolyte; ionic conduction; solid-state ionics; poly-2-vinylpyridine; transport number.

\section{Introduction}

Polymer electrolytes or polyelectrolytes are complexes of electron donor polymers with various inorganic or organic salts or acids. ${ }^{1}$ Solid state polymer electrolytes are gaining importance as ionics in solid state electrochemistry during last three decades. These are used in electrochemical devices such as high energy density batteries, ${ }^{2,3}$ measuring systems and display devices. These are also used in solid oxide fuel cells, chemical sensors, capacitors, etc. The main advantages of polymer electrolytes are their mechanical properties, easy fabrication into thin films of desired sizes and their ability to form proper electrode-electrolyte contact. Solid-state ionics materials are highly used in resistive random access memory devices and energy storage devices. Electrolytes, polyelectrolytes or their complexes in solid or liquid state can be used for electroplating and water purification. The merits of solid-state electrolytes are that these are much more reliable and long lasting materials under rough conditions of uses than comparable devices with liquid electrolytes.

The first solvent-free polyelectrolyte reported is the poly(ethylene oxide) (PEO)-based complex with alkali metal salts. $^{2,4,5}$ Some of the important classes of polymer electrolytes are PEO, polypropylene oxide, polyvinyl alcohol doped with alkali-metal salts or metal salts or acids. ${ }^{2,6,7}$

Measurement of conductivity in solid state shows that the ionic character is more prominent in alkali metal derivatives

\footnotetext{
*Author for correspondence (dr.neelot@gmail.com)
}

of polyvinyl borate (PVBO-M) than that of polyvinyl borate (PVBO). ${ }^{8}$ Similarly, the ionic conductivity of poly-2-vinyl pyridine hydrochloride $(\mathrm{P}-2 \mathrm{VP}-\mathrm{HCl})$ is found to be more than that of poly-2-vinyl pyridine (P-2VP). Poly-2-vinyl pyridine hydroiodide (P-2VP-HI ${ }^{9}$ was synthesized and found that its ionic conductivity increases very appreciably and P-2VP-HI proved to be a very good polyelectrolyte. Similar result has been found by synthesizing the salt of $\mathrm{HNO}_{3}$ with P-2VP. ${ }^{9}$ For imparting better polyelectrolyte character it is assumed that conducting powers of these materials differ widely when their conducting ion partners are altered.

The synthesis of the polyelectrolyte salts of P-2VP with some weak organic acids like crotonic acid (CrotA) and vinyl acetic acid (VAA) are not reported in literature. Moreover, mineral acids are toxic and corrosive. Therefore, an attempt has been made in our laboratory to synthesize the solid-state polyelectrolytes of P-2VP with CrotA and VAA and to characterize them with the help of Fourier transform infrared spectroscopy (FT-IR), proton nuclear magnetic resonance spectroscopy $\left({ }^{1} \mathrm{H} \mathrm{NMR}\right), \mathrm{X}$-ray diffraction (XRD) analysis, thermogravimetric analysis (TGA) and differential scanning calorimetry (DSC) techniques and then study their conductivities and ionic properties in solid state. Alternating current $(\mathrm{AC})$ conductivities are measured with the help of complex impedance/admittance plot and transport numbers are determined by the standard Wagner polarization technique to find out the degree of conduction and the ratio of ionic to electronic conduction, respectively.

In the present work, to synthesize the polyelectrolyte, unlike PEO complexes with alkali metals or other salts, no external salt is used; instead, the virgin polymer and its salts act as a polyelectrolyte. 


\section{Experimental}

\subsection{Materials}

2-Vinylpyridine (2-VP; Alfa Aesar), acetone (Ranbaxy), hydrochloric acid (Ranbaxy), carbon tetrachloride $\left(\mathrm{CCl}_{4}\right)$ (Ranbaxy), chloroform (SRL), potassium bromide $(\mathrm{KBr})$ (Merck), trans-CrotA (Alfa Aesar) and VAA (Alfa Aesar) were used without further purification.

\subsection{Preparation of $P-2 V P$}

$2-\mathrm{VP}$ is thermally polymerized in vacuum at $60^{\circ} \mathrm{C}$ for 1 day. The polymer so formed was dissolved in benzene and then precipitated in water. Reprecipitation was done for 3-4 times to remove the last traces of the monomer. The polymer so obtained was then dried in an oven at $60^{\circ} \mathrm{C}$ for $12 \mathrm{~h} .^{7,10}$

\subsection{Preparation of $P-2$ VP-CrotA salt}

A concentrated solution of P-2VP in chloroform was treated with CrotA in chloroform to dissolve at room temperature for 1 day. It was then precipitated by $\mathrm{CCl}_{4}$. The salt so formed was dissolved in water and then reprecipitated by $\mathrm{CCl}_{4}$ to get it in pure form. It was stored in a desiccator.

\subsection{Preparation of P-2VP-VAA salt}

A concentrated solution of P-2VP in chloroform was treated with VAA at room temperature for 1 day. The salt was precipitated by $\mathrm{CCl}_{4}$. It was dissolved in water and then reprecipitated by $\mathrm{CCl}_{4}$ to get it in pure form and stored in a desiccator.

\section{Measurements}

FT-IR spectra were recorded using NICOLET 6700 spectrophotometer in the range of $400-4000 \mathrm{~cm}^{-1}$. The spectra were recorded in transmission mode over 32 scans. Crystalline samples, for FT-IR measurements, were prepared in the form of pellets with the help of a $\mathrm{KBr}$ press by mixing about $20 \mathrm{mg}$ of IR spectroscopic grade $\mathrm{KBr}$ with about 2 mg of dried samples. For amorphous polymers, pellets were prepared by $20 \mathrm{mg}$ of $\mathrm{KBr}$ and poured over it one drop of the solution of amorphous polymer in chloroform and kept in a desiccators to dry and evaporate the solvent. The 300 $\mathrm{MHz}$ proton NMR spectra were recorded with a BRUKER DPX-300 NMR spectrophotometer using deuterated chloroform $\left(\mathrm{CDCl}_{3}\right)$ as solvent. The X-ray diffraction pattern was collected on a Bruker D8 Advance diffractometer using $\mathrm{Cu}$ $\mathrm{K} \alpha(\lambda=1.54 \AA)$ as the incident radiation operating at 40 $\mathrm{kV}$ and $40 \mathrm{~mA}$. Thermo-gravimetric analysis was performed by using Perkin Elmer TGA 4000 at a heating rate of $10^{\circ} \mathrm{C}$ $\mathrm{min}^{-1}$ with a nitrogen flow rate of $20 \mathrm{ml} \mathrm{min}^{-1}$. Differential scanning calorimetry was performed by using Perkin Elmer DSC 6000 over a temperature range of $30-400^{\circ} \mathrm{C}$.

AC conductivity measurements were carried out by HIOKI 3522-50 LCR HiTester frequency response analyzer by applying frequency from $1 \mathrm{~Hz}$ to $100 \mathrm{kHz}$ keeping the voltage at $0.1 \mathrm{~V}$. The temperature range applied was from 30 to $90^{\circ} \mathrm{C}$. The polymer and the polylectrolyte samples were taken in the form of thin films sandwiched between two symmetric stainless steel (SS) electrodes. The geometry of the cell for the measurement of conductivity was SSIpolymer filmISS. The thicknesses of the films used for AC conductivity measurement were $0.052,0.053$ and $0.023 \mathrm{~cm}$ for P-2VP, P-2VP-CrotA and P-2VP-VAA, respectively. The experiment was carried out under a relative humidity of $72 \%$. Before the measurement, all samples were kept at $60^{\circ} \mathrm{C}$ for $1 \mathrm{~h}$ to ascertain the interface contact between the electrolyte film and the electrodes and for recording the measurements each sample was kept at each temperature at least for $10 \mathrm{~min}$ to attain the thermal equilibrium.

Transport number gives the fraction of current carried by anions, cations or electrons in the material in terms of total conductivity. ${ }^{11}$ Transport number study of the salts was done to determine the conduction behaviour. The total ionic transport number, $t_{\text {ion }}$, was evaluated by the standard Wagner polymerization technique. ${ }^{12}$ The cell SSIpolymer filmISS [SS stands for stainless steel] was polarized by a step potential (about $1.0 \mathrm{~V}$ ) and the resulting potentiometric current was monitored as a function of time. The SS acted as blocking electrodes for the above cell. The $t_{\text {ion }}$ was evaluated using the formula

$$
t_{\text {ion }}=\left(i_{\mathrm{T}}-i_{\mathrm{e}}\right) / i_{\mathrm{T}},
$$

where $i_{\mathrm{T}}$ and $i_{\mathrm{e}}$ are the total and the residual current, respectively.

\section{Results and discussion}

\subsection{Reaction schemes}

The probable reactions of P-2VP with CrotA and VAA may be as follows:

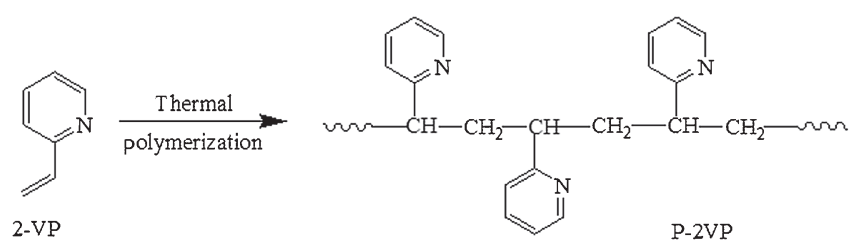

2-VP

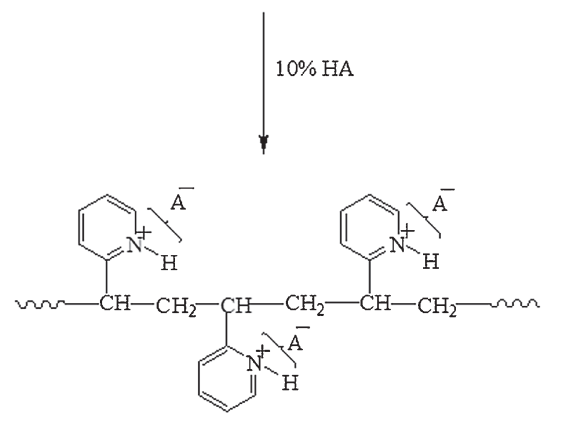

P-2VP-CrotA or P-2VP-VAA 


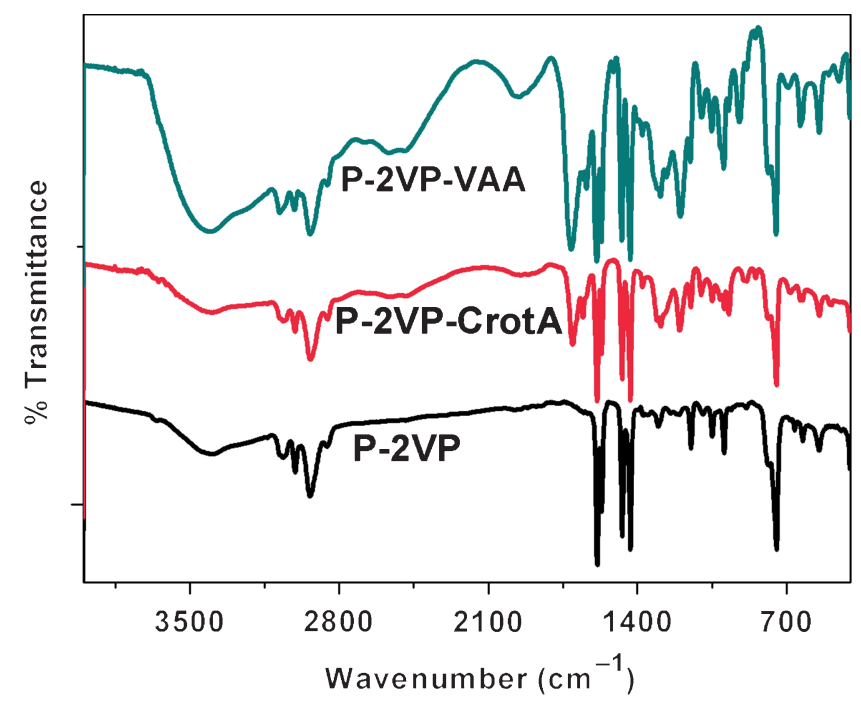

Figure 1. FT-IR spectra of P-2VP, P-2VP-CrotA and P-2VP-VAA.

where $\mathrm{A}^{-}$refers to $\mathrm{CH}_{3} \mathrm{CH}=\mathrm{CHCOO}^{-}$and $\mathrm{CH}_{2}=\mathrm{CHCH}_{2}$ $\mathrm{COO}^{-}$, the negative counter ion of CrotA and VAA, respectively.

2-VP is a basic molecule due to the presence of the electron withdrawing pyridinyl group. In P-2VP, the polymeric character predominates. In the presence of an acid, the nitrogen lone pair of electrons easily forms a new $\mathrm{N}-\mathrm{H}$ bond with $\mathrm{O}-\mathrm{H}$ proton of the acid and behaves like a positive ion. This ion then forms stable salt with the negative counter ion of the acid. In this process, the ionic character of the polymer salt increases resulting in good polyelectrolyte.

\subsection{Characterization}

4.2a FT-IR analysis: The FT-IR spectra of P-2VP, P-2VPCrotA and P-2VP-VAA are shown in figure 1. The peaks observed in all the three spectra at 3061,3007 and $2931 \mathrm{~cm}^{-1}$ are due to $\mathrm{C}-\mathrm{H}$ stretching absorption. The ring stretching vibrations $\mathrm{C}=\mathrm{C}$ and $\mathrm{C}=\mathrm{N}$ occur at 1591,1473 and $1425 \mathrm{~cm}^{-1}$. The band at $751 \mathrm{~cm}^{-1}$ is due to $\mathrm{C}-\mathrm{H}$ out-of-plane bending vibration. ${ }^{13}$ The formation of the salts P-2VP-CrotA and $\mathrm{P}-2 \mathrm{VP}-\mathrm{VAA}$ are confirmed from the appearance of the $\mathrm{C}=\mathrm{O}$ stretching band at $1714 \mathrm{~cm}^{-1}$ in both the spectra (b) and (c). The spectrum (b) shows a distinct peak at $1652 \mathrm{~cm}^{-1}$ and the spectrum (c) shows a distinct peak at $1638 \mathrm{~cm}^{-1}$ which are absent in the spectrum (a) are due to the presence of the carboxylate anion $\left(\mathrm{COO}^{-}\right)$and also $\mathrm{C}=\mathrm{C}$ stretching vibration. ${ }^{13}$

4.2b ${ }^{1} H$ NMR analysis: $\quad$ The ${ }^{1} \mathrm{H}$ NMR spectrum of P-2VP (figure 2a) indicates that the two signals observed at 1.79 and $2.49 \mathrm{ppm}$ are due to $-\mathrm{CH}_{2}-$ and $-\mathrm{CH}-$ proton, respectively. The signals ranging from 6.23 to $7.36 \mathrm{ppm}$ are due to the protons of pyridine molecule. ${ }^{14}$

The ${ }^{1} \mathrm{H}$ NMR data of P-2VP-CrotA (figure $2 b$ ) indicate that the signal observed at $1.7-1.9 \mathrm{ppm}$ is due to $-\mathrm{CH}_{3}$

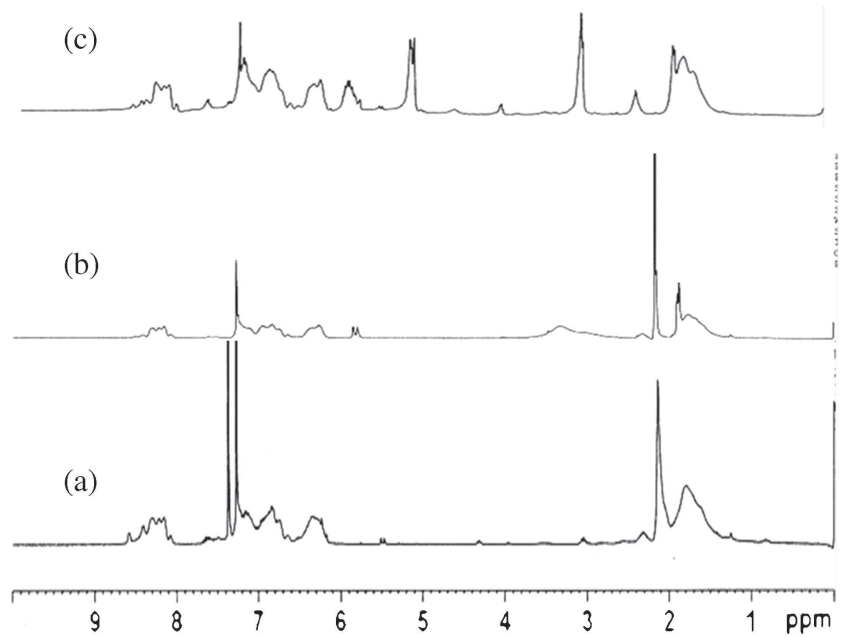

Figure 2. ${ }^{1} \mathrm{H}$ NMR spectrum of (a) P-2VP, (b) P-2VP-CrotA and (c) P-2VP-VAA.

protons. The other peak at $2.1 \mathrm{ppm}$ is due to $\mathrm{CH}_{2}$ protons. The signals observed at 3.3 and $5.8 \mathrm{ppm}$ are due to the presence of $\mathrm{CH}$ protons. The signals ranging from 6.2 to 7.2 $\mathrm{ppm}$ are due to pyridine molecule. The addition of CrotA to $\mathrm{P}-2 \mathrm{VP}$ is confirmed by the signal in between 8 and 9 ppm. ${ }^{10}$

The ${ }^{1} \mathrm{H}$ NMR data of P-2VP-VAA (figure 2c) indicate that the signal from 1.6 to $1.8 \mathrm{ppm}$ is due to the $-\mathrm{CH}_{2}-$ (methylene proton in the vinyl chain) protons and the signal from 2.3 to $2.9 \mathrm{ppm}$ is due to $-\mathrm{CH}_{2}$ - (connected to the $\mathrm{COO}^{-}$group). On the other hand, the signal found at $3.0 \mathrm{ppm}$ is due to the $-\mathrm{CH}-$ protons. The signal from 5.0 to $5.1 \mathrm{ppm}$ appears for $=\mathrm{CH}_{2}$ protons and the signal appears from 5.8 to $5.9 \mathrm{ppm}$ for $-\mathrm{CH}=$ protons. The broad signal ranging from 6.2 to $7.6 \mathrm{ppm}$ appears due to pyridine molecule. A new signal which is absent in the reactants appears from 8.0 to $8.4 \mathrm{ppm}$, indicating the formation of a new salt P-2VP-VAA. ${ }^{6}$

4.2c XRD analysis: The $\mathrm{X}$-ray diffraction patterns of $\mathrm{P}-2 \mathrm{VP}$, $\mathrm{P}-2 \mathrm{VP}-\mathrm{CrotA}$ and P-2VP-VAA are shown in figure 3. The results show that all these newly synthesized salts along with $\mathrm{P}-2 \mathrm{VP}$ are all amorphous in nature although CrotA itself is a crystalline solid. The amorphous nature indicates the segmental motion of the polyelectrolytes which is essential for ionic conduction.

4.2d Thermogravimetric analysis: The TGA thermogram of P-2VP, P-2VP-CrotA and P-2VP-VAA are shown in figure 4. The TGA thermogram of P-2VP shows that upto $124^{\circ} \mathrm{C}, 4.1 \%$ weight is lost which is generally moisture and upto $361^{\circ} \mathrm{C}$ another $20.4 \%$ weight is lost. The major weight loss occurs at $422^{\circ} \mathrm{C}$ which is another $57.4 \%$. The polymer is completely decomposed at $633^{\circ} \mathrm{C}$.

The thermogram of P-2VP-CrotA indicates that up to $165^{\circ} \mathrm{C}, 10 \%$ weight was lost which was due to removal of absorbed moisture and entrapped solvent molecules. From $165^{\circ} \mathrm{C}$, 2nd weight loss was occurred gradually. The major weight loss occurred was found to be at $350^{\circ} \mathrm{C}$. From 350 to 


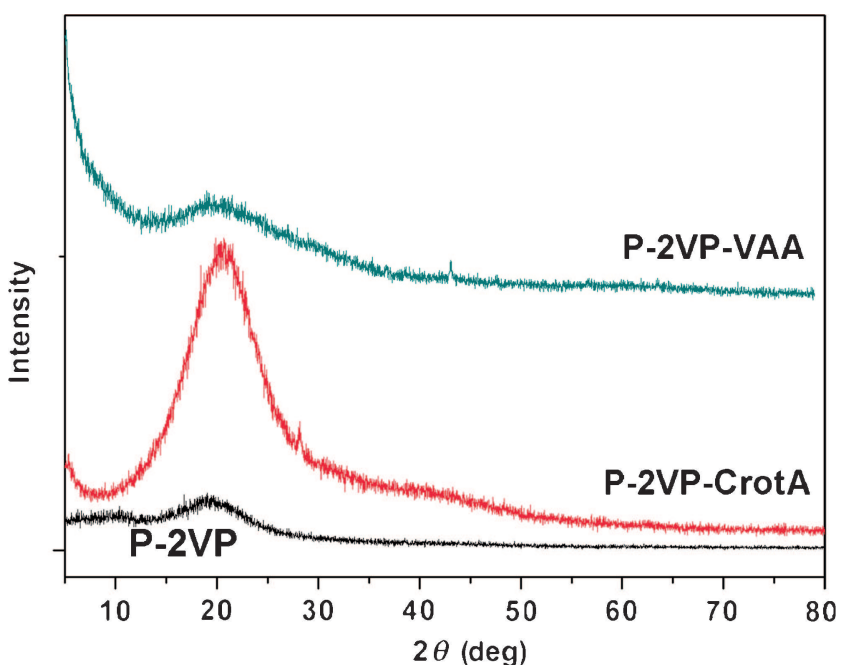

Figure 3. XRD of $\mathrm{P}-2 \mathrm{VP}, \mathrm{P}-2 \mathrm{VP}-\mathrm{Crot} \mathrm{A}$ and $\mathrm{P}-2 \mathrm{VP}-\mathrm{VAA}$.

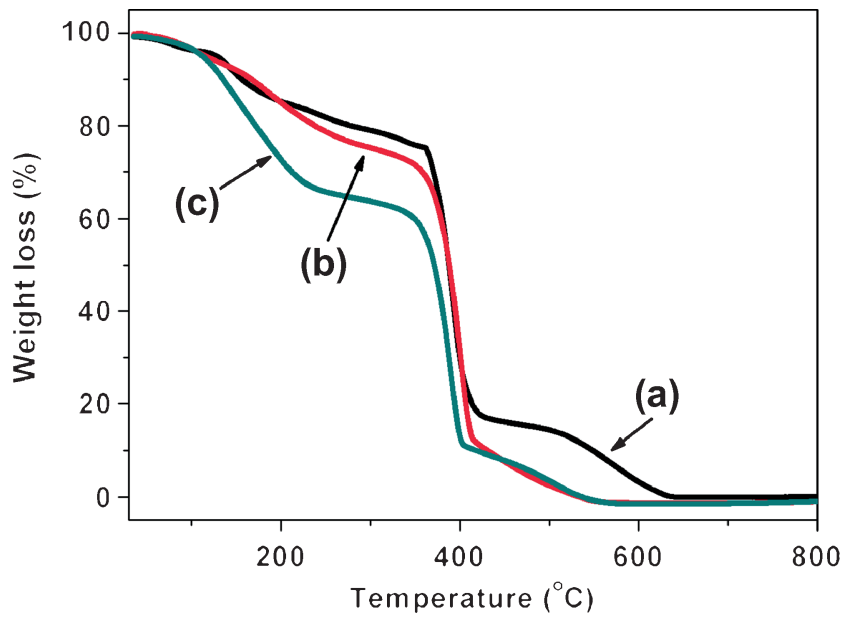

Figure 4. TGA of (a) P-2VP, (b) P-2VP-CrotA and (c) P-2VPVAA.

$414^{\circ} \mathrm{C}$ the percentage of weight loss was $66 \%$ This weight loss might be due to loss of pyridine and $\mathrm{CO}_{2}$ molecules.

The thermogram of P-2VP-VAA shows the initial loss of mass $(29 \%)$ from $110^{\circ} \mathrm{C}$ upto $232^{\circ} \mathrm{C}$ was due to removal of absorbed moisture and entrapped solvent molecules. The decomposition temperature for the first major step was found to be at $350^{\circ} \mathrm{C}$. From 350 to $402^{\circ} \mathrm{C}$ the percentage of weight loss was $56 \%$. The complete degradation of the polymer salt occurred at $530^{\circ} \mathrm{C}$.

4.2e DSC analysis: The DSC thermograms of P-2VP, P-2VP-CrotA and P-2VP-VAA are shown in figure 5. From the thermogram, the glass transition temperature $\left(T_{\mathrm{g}}\right)$ of P-2VP was found to be $85^{\circ} \mathrm{C}$. The $T_{\mathrm{g}}$ of P-2VP-CrotA and $\mathrm{P}-2 \mathrm{VP}-\mathrm{VAA}$ were found as 36 and $35^{\circ} \mathrm{C}$, respectively, from the DSC curves. It is observed that the addition of CrotA or VAA causes obvious decrease in $T_{\mathrm{g}}$ of the polyelectrolyte. This is probably because of the resulting new

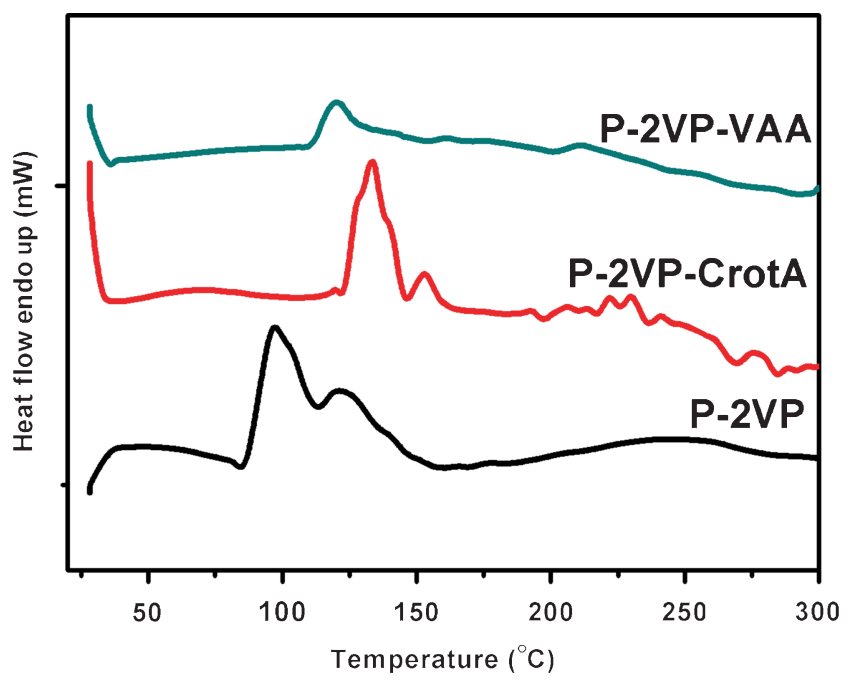

Figure 5. DSC of P-2VP, P-2VP-CrotA and P-2VP-VAA.

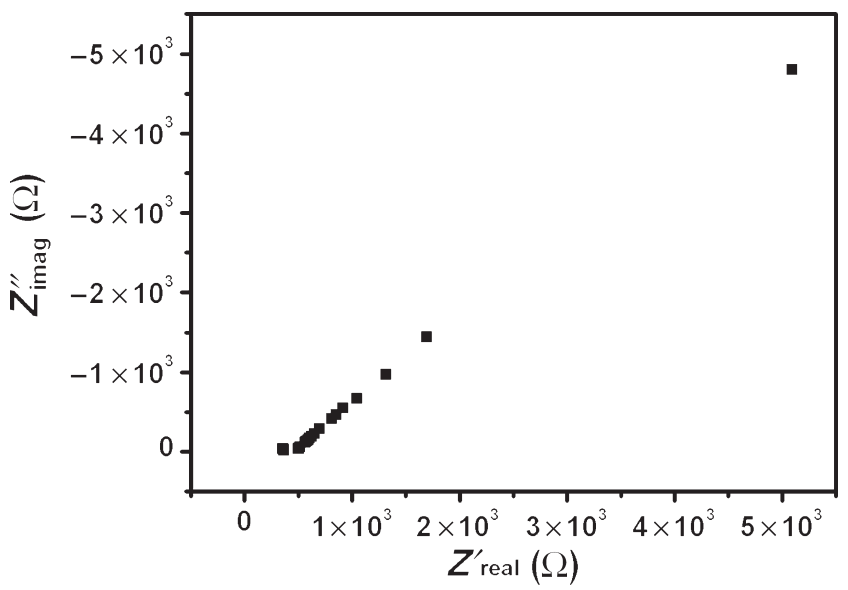

Figure 6. Complex impedance plot of P-2VP-VAA at $80^{\circ} \mathrm{C}$.

$\mathrm{N}-\mathrm{H}$ bond between the polymer and the acid due to the for mation of the salt in the form of pyridinium ion and the counter ion of the acid which facilitate the migration of the ions and thereby increasing the flexibility of the polymer. The increasing flexibility of a polymer increases its ionic conductivity. ${ }^{15}$

\subsection{AC conductivity measurements}

The conductivities of the polymer and the polyelectrolytes are measured by AC impedance spectroscopy. The impedance of the polymer and its two salts were recorded by the HIOKI 3522-50 LCR HiTester frequency response analyzer at seven different temperatures of $10^{\circ} \mathrm{C}$ interval ranging from 30 to $90^{\circ} \mathrm{C}$. Therefore, for each sample, there obtained seven complex-impedance plots. The complex-impedance plots, i.e., $Z \cos \theta$ vs. $Z \sin \theta$ plots where $Z \cos \theta$ is the real part and $Z \sin \theta$ is the imaginary part denoted as $Z_{\text {real }}^{\prime}$ and $Z_{\text {imag }}^{\prime \prime}$, respectively. In this way for the three samples there are a total of 21 complex-impedance plots. For example, figure 6 shows the typical impedance plot of P-2VP-VAA 
Table 1. Conductivity values of P-2VP and its salts at different temperatures.

\begin{tabular}{lccc}
\hline & \multicolumn{3}{c}{ Conductivity $\left(\mathrm{S} \mathrm{cm}^{-1}\right)$} \\
\cline { 2 - 4 } Temperature $\left({ }^{\circ} \mathrm{C}\right)$ & $\mathrm{P}-2 \mathrm{VP}$ & $\mathrm{P}-2$ VP-CrotA & P-2VP-VAA \\
\hline 30 & $1.99 \times 10^{-8}$ & $1.81 \times 10^{-7}$ & $5.13 \times 10^{-6}$ \\
40 & $2.12 \times 10^{-8}$ & $5.44 \times 10^{-7}$ & $1.13 \times 10^{-5}$ \\
50 & $2.22 \times 10^{-8}$ & $1.18 \times 10^{-6}$ & $1.56 \times 10^{-5}$ \\
60 & $3.01 \times 10^{-8}$ & $2.19 \times 10^{-6}$ & $2.36 \times 10^{-5}$ \\
70 & $3.31 \times 10^{-8}$ & $3.70 \times 10^{-6}$ & $2.89 \times 10^{-5}$ \\
80 & $4.53 \times 10^{-8}$ & $6.34 \times 10^{-6}$ & $3.68 \times 10^{-5}$ \\
90 & $7.15 \times 10^{-8}$ & $8.74 \times 10^{-6}$ & $6.90 \times 10^{-5}$ \\
\hline
\end{tabular}

at $80^{\circ} \mathrm{C}$. The point of intersection of the two semicircles of a complex-impedance plot coincides with the bulk resistance $\left(R_{\mathrm{b}}\right)$ of the polyelectrolyte. The small semicircle corresponds to the medium-high frequencies and the large semicircle corresponds to the low frequencies. In our experiment, the semicircle at high frequency range is not completely observed, which may be concluded that the frequencies we have selected were not high enough. ${ }^{16}$

After determining the value of $R_{\mathrm{b}}$, the ionic conductivity $(\sigma)$ is calculated from the relation ${ }^{17}$

$$
\sigma=t / R_{\mathrm{b}} A
$$

where $t$ is the thickness of the polymer film and $A$ the area of the film.

Table 1 shows the values of bulk conductivity of the polymer and the polyelectrolytes at different temperatures. It is found that the conductivity of each sample increases with the increase of temperature. The conductivities of P-2VP-VAA and $\mathrm{P}-2 \mathrm{VP}-\mathrm{CrotA}$ are higher than that of $\mathrm{P}-2 \mathrm{VP}$ at the whole temperature range. In case of $\mathrm{P}-2 \mathrm{VP}-\mathrm{CrotA}$, there is a onefold increase in conductivity at $30^{\circ} \mathrm{C}$ and two-fold increase in conductivity at $90^{\circ} \mathrm{C}$. On the other hand, there is a twofold increase in conductivity at $30^{\circ} \mathrm{C}$ and three-fold increase in conductivity at $90^{\circ} \mathrm{C}$ in case of P-2VP-VAA.

The activation energies $\left(E_{\mathrm{a}}\right)$ of various reactions are calculated from the plot $\log (\sigma T)$ vs. 1000/T using the Arrhenius relation (equation 3 )

$$
\sigma=\sigma_{\mathrm{o}} \exp \left(-E_{\mathrm{a}} / k T\right)
$$

where $\sigma_{\mathrm{o}}$ is the pre-exponential factor, $k$ the Boltzmann constant and $T$ the absolute temperature

The activation energies computed from Arrhenius plots (figure 7) are $0.202,0.6128$ and $0.4105 \mathrm{eV}$ for conduction of P-2VP, P-2VP-CrotA and P-2VP-VAA, respectively. The activation energy values of P-2VP-CrotA and P-2VP-VAA are much higher than the activation energy value of P-2VP. The higher value of activation energy indicates the higher value of percentage of ionic character because higher value of activation energy is necessary for ionic conduction. ${ }^{18,19}$

From the Arrhenius plots it is also seen that two salts, i.e., P-2VP-VAA and P-2VP-CrotA have more conductivity

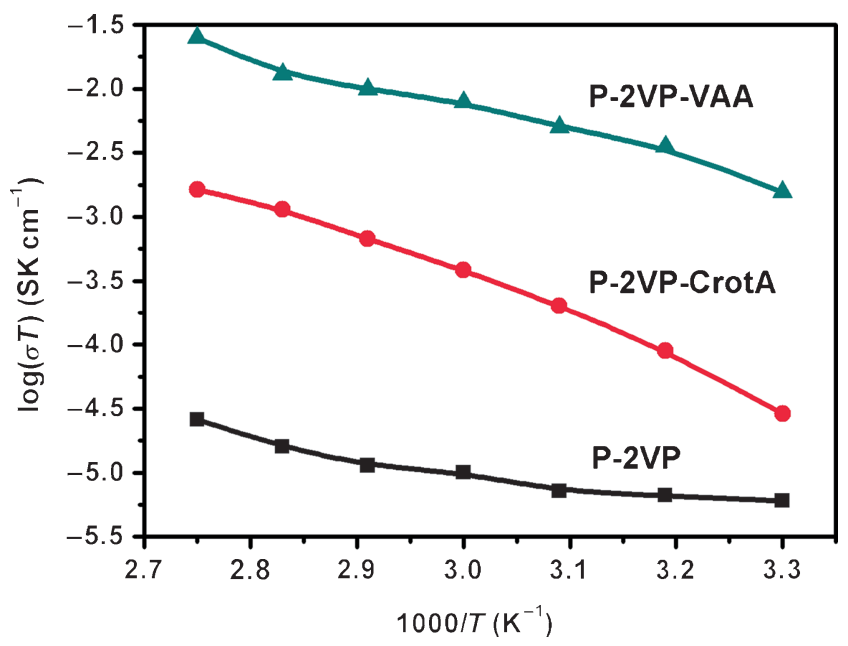

Figure 7. $\log (\sigma T)$ vs. $1000 / T$ plot for P-2VP, P-2VP-CrotA and P-2VP-VAA.

than P-2VP. The reason for higher conductivity of P-2VPVAA may be due to the presence of the vinyl group at the end of the chain of the acid. Because of the presence of this group the carboxylic acid protons acquires more acidic character. Hence the conductivity of P-2VP-VAA is very much higher than the conductivity of P-2VP. On the other hand, in CrotA there is a methyl group. We know that methyl group is an electron donating group. So, it is less easy to release the proton from CrotA than VAA. That is why the conductivity of P-2VP-VAA is higher than the conductivity of P-2VPCrotA. But the conductivities of both these salts are very much higher than the conductivities of the virgin polymer P-2VP and can be used as good polyelectrolytes.

\subsection{Transport number measurements}

The total ionic transport numbers of the polyelectrolytes were determined by the standard Wagner polarization technique the experimental setup of which is given in figure $8 .^{8,20}$ When a voltage is applied, ion migrations take place until a steady state is reached. At this state, the cell is polarized and any residual current flows because of electron migration across the electrode-electrolyte interfaces. In case of ionic samples, the current passing through blocking electrodes falls rapidly with time. In our experiment, the polymer P-2VP shows slight fall of current with time when a voltage is applied whereas the polymer salts P-2VP-CrotA and P-2VP-VAA show a rapid fall of current as time passes when the voltage is applied. The plots of current vs. time for each sample are shown in figure 9a which reveals that $\mathrm{P}-2 \mathrm{VP}$ shows mixed ionic and electronic conductivity and in case of P-2VP-CrotA and P-2VP-VAA the major mechanism for conduction is ionic (figure 9b). From the transport number studies it is found that the total ionic transport number for P-2VP, P-2VP-CrotA and P-2VP-VAA are 0.76, 0.86 and 0.96 , respectively. The experiments were repeated thrice. 


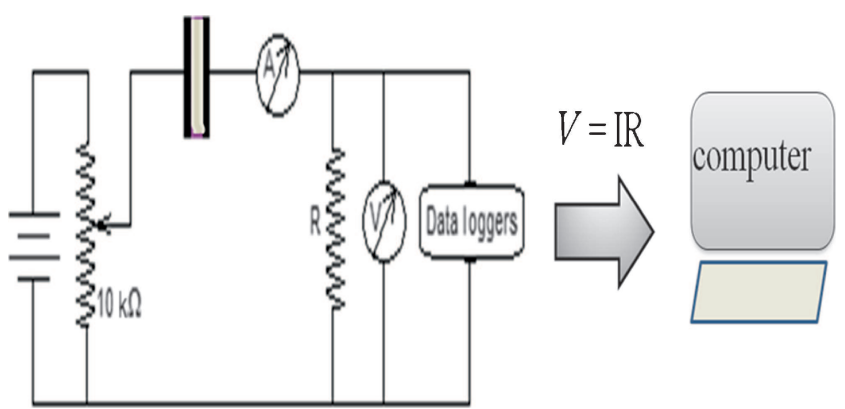

Figure 8. Schematic representation of cell circuit used to determine the transport number.

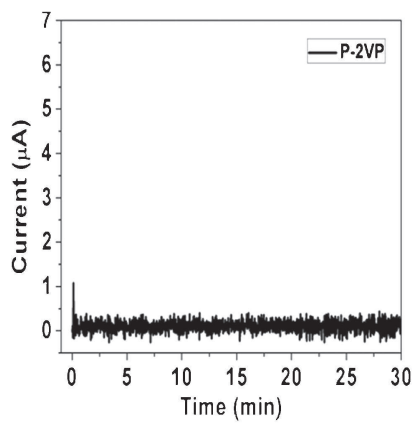

(a)

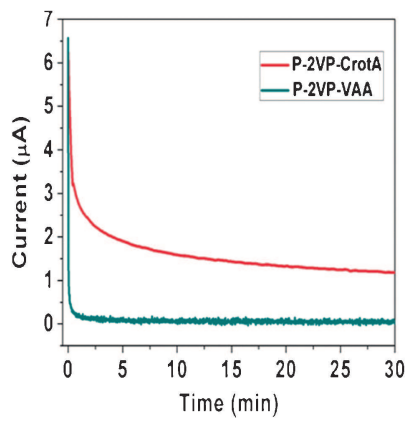

(b)

Figure 9. Time vs. current plots of (a) P-2VP and (b) P-2VPCrotA and P-2VP-VAA.

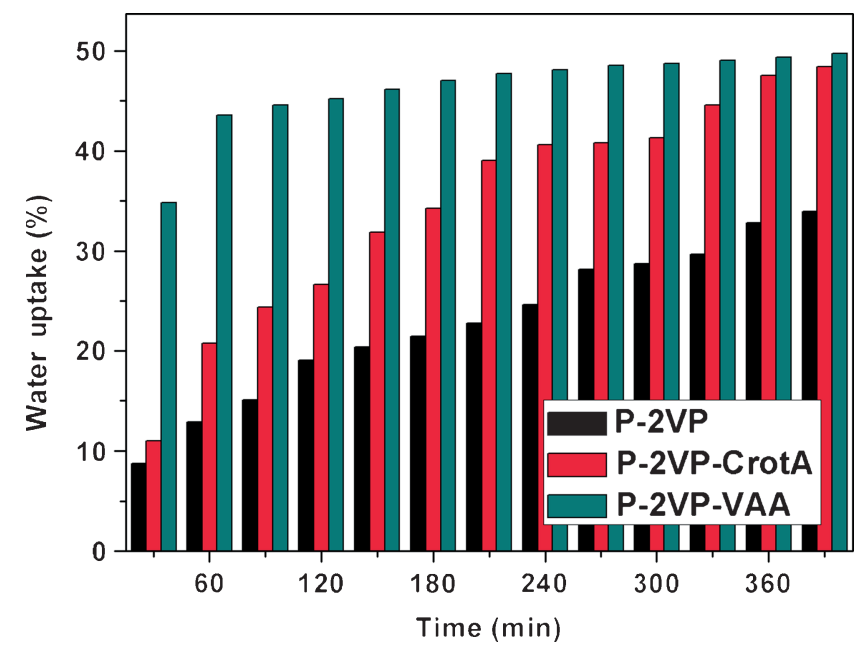

Figure 10. Percentage of water uptake of the polymer and the polyelectrolytes.

4.4a Water uptake property: The water uptake properties are calculated as percentage by using the following relation: ${ }^{21}$

$$
\text { Water uptake }(\%)=\left\{\left(W_{\mathrm{w}}-W_{\mathrm{d}}\right) / W_{\mathrm{d}}\right\} 100,
$$

where $W_{\mathrm{w}}$ and $W_{\mathrm{d}}$ are the weights of wet and dry samples, respectively.

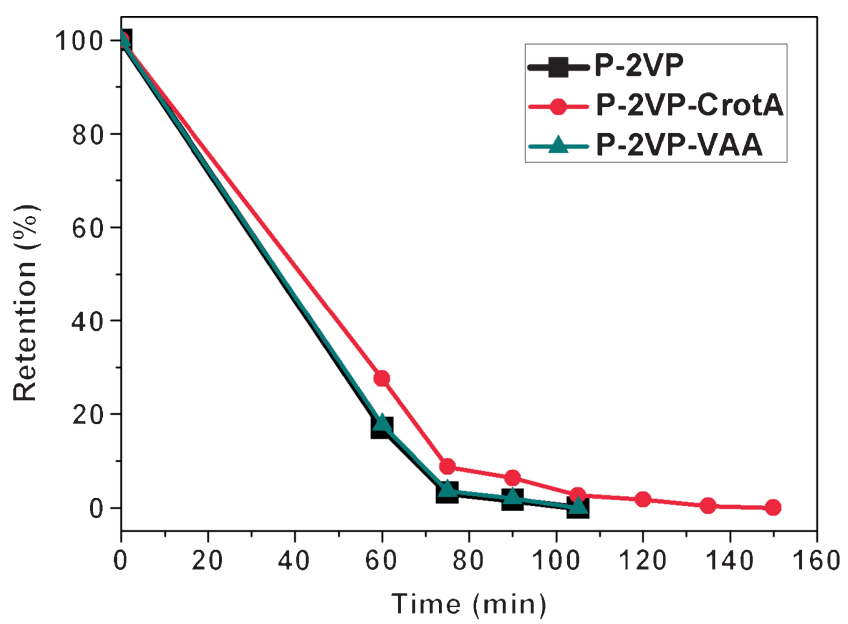

Figure 11. Water retention properties of the polymer and the polyelectrolytes.

The percentage of water uptake of the polymer and the polyelectrolytes were measured at room temperature $\left(25^{\circ} \mathrm{C}\right)$. To carry out this experiment, first the weights of the dry polymer and the polyelectrolytes were recorded. Then the samples were immersed separately in beakers containing water. After different intervals of time, the samples were taken out of the beakers blotted quickly to remove excess water and again weighed. The saturation time for all samples was found at about $6 \mathrm{~h}$. The bar diagram of the percentage of water uptake of all the samples is shown in figure 10 .

The retention time was calculated for all the samples at room temperature (figure 11). It was observed that the polymer as well as the polyelectrolytes required almost two to two and half hours to get back its original form.

\section{Conclusion}

In conclusion, we have improved the conducting properties of P-2VP by preparing its salts with CrotA and VAA. These materials can be considered as polyelectrolytes. Incorporation of weak acids also can increase the conductivities of such materials which is the major significance of this work. The polymer and the prepared salts peculiarly show amorphous character although one acid, i.e., CrotA is crystalline. The amorphous character corresponds to the increase in conductivity of the polyelectrolytes by increasing its segmental motions. The salts are thermally stable upto $110^{\circ} \mathrm{C}$ and can be used as good polyelectrolytes in solid state. The total ionic transport number of $\mathrm{P}-2 \mathrm{VP}$ is found to be increased after the formation of polyelectrolyte salts with the acids. P-2VP shows a mixed ionic and electronic conduction. But for the salts with CrotA and VAA its conductivity increases up to about two- and three-fold, respectively. This is due to the production of positive and negative ions in the form of salts. Thus these salts may be used as intrinsic conducting polymers in solid state. 


\section{References}

1. Bruce P G 1995 Solid state electrochemistry (Cambridge: Cambridge University Press) p 96

2. Armand M B, Chabagno J M and Duclot M J 1979 In Fast ion transport in solids (eds) P Vashishta, J N Mundy and G K Shenoy (Amsterdam: North-Holland Publ Co.) p 131

3. Fawcett A H 1987 In Encyclopedia of polymer science and engineering (eds) J Korschwitz and J Korschwitz (New York: Wiley) 2nd ed, p 10

4. Fenton D E, Parker J M and Wright P V 1973 Polymer 14589

5. Wright P V 1975 Br. Polym. J. 7319

6. Sarma N S and Dass N N 2001 Mater. Sci. Eng. B 7978

7. Sarma N S, Dutta A and Dass N N 2003 Eur. Polym. J. 1071

8. Chetri P, Dass N N and Sarma N S 2007 Mater. Sci. Eng. B 139261

9. Chetri P, Dass N N and Sarma N S 2006 J. Polym. Mater. 23 397

10. Sarma N S and Dass N N 2002 J. Polym. Mater. 19179

11. Wagner J B and Wagner C 1957 J. Chem. Phys. 261597
12. Hashmi S A and Chandra S 1995 Mater. Sci. Eng. B 3418

13. Silverstein R M, Bassler G C and Morrill T C 1991 Spectrometric identification of organic compounds (United States of America: John Wiley \& Sons, Inc.) 5th ed, p 117

14. Dyer J R 1991 Application of absorption spectroscopy of organic compounds (United States of America: Prentice-Hall International, Inc.) 8th ed, p 38

15. Chakrabarty A, Filler R and Mandal B K 2008 J. Solid State Electrochem. 12269

16. Chun-yue P, Qing F, Li-jun W, Qian Z and Meng C 2007 J. Cent. South Univ. Technol. 14348

17. Li W 2009 Development and understanding of new membranes based on aromatic polymers and heterocycles for fuel cells (University of Texas) p 33

18. Maurya K K, Bhattacharya B and Chandra S 1995 Phys. Status Solidi (a) 147347

19. Mikhailenko S D, Kaliaguine S and Moffat J B 1997 Solid State Ion 99285

20. Munstedt H 1986 Polymer 27899

21. Son J H, Kang Y S and Won J 2006 J. Membr. Sci. 281345 